\title{
ANTIOXIDANT CAPACITY OF SEMINAL PLASMA MEASURED BY TAS RANDOX ${ }^{\circledR}$
} \author{
Milena Krskova ${ }^{\mathrm{d}}$, Ivana Oborna ${ }^{\mathrm{a} *}$ \\ a Department of Obstetrics and Gynecology, University Hospital, Olomouc, Czech Republic \\ ${ }^{b}$ Department of Biology, Faculty of Medicine and Dentistry, Palacky University, Olomouc \\ c Department of Biochemistry, University Hospital Olomouc \\ ${ }^{d}$ Palacky University Computer Centre, Olomouc \\ e-mail: obornai@fnol.cz
}

Helena Fingerova $^{\mathrm{a}}$, Jiri Novotny ${ }^{\mathrm{b}}$, Jiri Barborik ${ }^{\mathrm{c}}$, Jana Brezinova ${ }^{\mathrm{a}}$, Magda Svobodova ${ }^{\mathrm{a}}$,

Received: March 22, 2007; Accepted: May 14, 2007

Key words: Oxidative stress/Antioxidant capacity/TAS Randox ${ }^{\circledR} /$ Seminal plasma/Blood serum/Male infertility

Background: An imbalance between reactive oxygen species (ROS) generation in sperm and antioxidant capacity of seminal plasma has been linked to male infertility. The antioxidant power of biological fluids can be evaluated either by measurement of individual antioxidants or total antioxidant capacity (TAC). The aim of this study was to assess whether TAS Randox ${ }^{\circledR}$ can also be used for seminal plasma antioxidant capacity estimation..

Material and Methods: Freshly thawed aliquots of seminal plasma and blood serum of 38 males from infertile couples and 24 healthy normospermic controls were simultaneously assayed using TAS Randox ${ }^{\circledR}$ reagents on the Hitachi Modular P800 Analyzer. Semen analysis was performed according to WHO guidelines. ROS in fresh sperm suspension in phosphate buffered saline was measured by chemiluminescence immediately after separation of seminal plasma.

Results: Semen analysis showed that in our study group only 14 males were normospermic and 24 males had mostly combined pathologies. The medians for ROS production were similar in both the study and the control groups (4850 and $5450 \mathrm{RLU} / \mathrm{min}$, resp). Seminal plasma TAS levels were significantly lower $(p<0.02)$ in the study group while blood serum TAS levels were similar in both groups. A significant positive correlation $(p<0.05)$ between TAS in seminal plasma and serum was found, seminal plasma levels being on average 1.4 times higher.

Conclusions: The TAS Randox ${ }^{\circledR}$ kit may be used for clinical studies intended to identify decreased antioxidant power in the seminal fluid of infertile men.

\section{INTRODUCTION}

Reactive oxygen species (ROS) have been implicated in the pathogenesis of many disease states, including infertility. Excessive ROS originating from immature sperm as well as from leukocytes can impair normal sperm function by peroxidation of unsaturated fatty acids in membrane of spermatozoa and by DNA fragmentation. On the other hand, a physiological level of ROS is crucial for sperm activation, capacitation and acrosomal reaction.

To protect spermatozoa from oxidative damage, seminal plasma is endowed with numerous enzymatic antioxidants (AOs) such as superoxide dismutase, catalase and glutathione peroxidase and a variety of non-enzymatic AOs such as ascorbate, urate, $\alpha$-tocopherol, pyruvate, glutathione, taurine and hypotaurine. The endogenous AOs may be further replenished by AOs derived from food, beverages, nutritional supplements and pharmaceuticals. The imbalance between excessive ROS generation and decreased antioxidant capacity leads to the condition called oxidative stress (OS) which has been linked to idiopathic male infertility and has become a rationale for antioxidant treatment ${ }^{1,2}$.
The antioxidant power of biological fluids can be evaluated either by quantification of individual AOs or by assessing their aggregate, cumulative action and synergic effect. This latter concept is known as the Total Antioxidant Capacity (TAC). The quantification of individual AOs (most often superoxide dismutase and catalase) is a complicated, expensive and time consuming task. Therefore the idea of a single measurement of total antioxidant capacity (TAC) is very attractive.

Numerous principles of assays of TAC in plants, food, beverages and body fluids have been published in the last 20 years. These include enhanced chemiluminescence (ECL) assays ${ }^{3}$, spectrophotometric methods such as FRAP assay ${ }^{4}$, CUPRAC assay ${ }^{5}$ or methods based on the formation of the $\mathrm{ABTS}^{+}$radical $^{6}$, fluorometric methods such as ORAC assay ${ }^{7}$ and electrochemical methods like coulometry ${ }^{8}$, voltammetry ${ }^{9}$ or electron spin resonance assay $^{10}$. Some of the above spectrophotometric and fluorometric assays are now commercially available.

The ECL method using luminol for measurement of non-enzymatic antioxidants was later used for seminal plasma by several investigators ${ }^{11,12}$. The ECL method is highly sensitive, but expensive and also time-consuming 
for use in clinical practice. A satisfactory correlation, albeit on a very small sample size, between the ECL method and the manual colorimetric method based on the formation of the radical ions, $\mathrm{ABTS}^{+}$has been reported ${ }^{13}$. This method has been commercialized by Randox Ltd. as the TAS (Total Antioxidant Status) Randox ${ }^{\circledR}$ kit. The aim of our study was to assess if the automated version of this kit designed for blood serum or plasma can be used for seminal plasma, too. An additional advantage of this would be the possibility of comparing TAS levels in blood with seminal plasma levels.

\section{MATERIALS AND METHODS}

The study group included samples of 38 males from infertile couples who registered for infertility treatment at the institutional IVF centre of the Department of Obstetrics and Gynecology, University Hospital in Olomouc from September 2005 to May 2006. Twenty

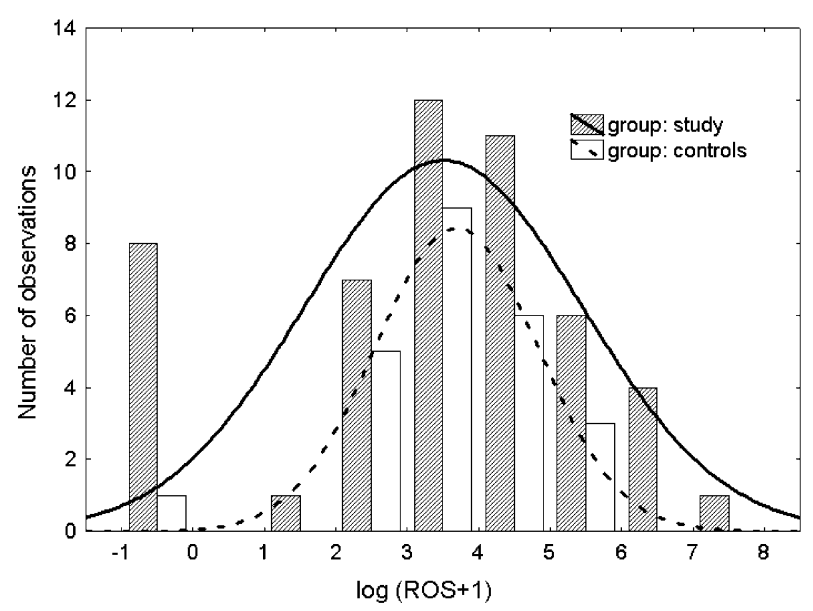

Fig 1. Overlap of the histograms of ROS levels in the control and study group.

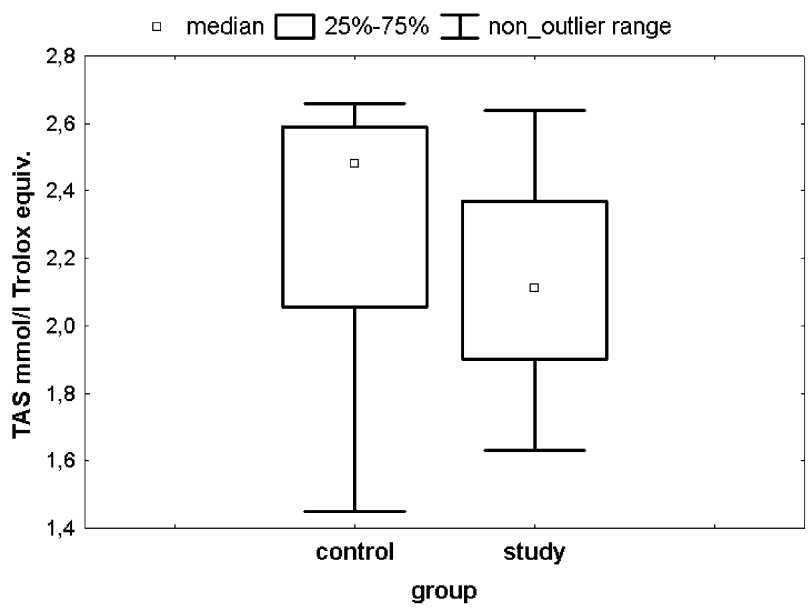

Fig 2. Significant difference between seminal plasma TAS levels in the control and study groups. four healthy volunteers or semen donors served as the control group where all samples had normal sperm parameters, i.e. sperm concentration more than 20 million $/ \mathrm{ml}$, motility more than $50 \%$ and more than $30 \%$ normal sperm morphology without leukocytospermia according to WHO guidelines ${ }^{14}$. All males signed written informed consent for recruitment into the study approved by the Ethics Board of the Medical Faculty of Palacky University in Olomouc.

Semen samples were collected by masturbation after three to four days of sexual abstinence. After liquefaction, semen analysis was performed according to WHO guidelines. After centrifugation of liquefied semen (at $300 \mathrm{~g}$ for $7 \mathrm{~min}$ ) the sperm pellet was washed and re-suspended in phosphate buffered saline (PBS). ROS production in sperm suspension was immediately determined by the ECL method described elsewhere ${ }^{15}$. Aliquots of seminal plasma were stored in liquid nitrogen. Fasting peripheral blood for determination of plasma TAS levels was placed in pre-cooled test tubes on the day of semen examination. Serum was immediately separated in refrigerated centrifuge and its aliquots were stored at $-20^{\circ} \mathrm{C}$ until further use. Freshly thawed batches of seminal plasma and blood serum were analyzed with the use of the TAS Randox ${ }^{\circledR}$ kit (Cat No. NX2332, Randox laboratories, Ltd, Crumlin, UK) on the Modular SVA Analyzer. Samples were transferred into primary cups and SVA Analyzer was programmed according to the recommendation of manufacturer. Results were expressed in $\mathrm{mM}$ of Trolox equivalents. The linearity of calibration extends to $2.5 \mathrm{mmol} / 1$ of Trolox. The reference range for human blood plasma is given by the manufacturer as $1.30-1.77 \mathrm{mmol} / 1$, no data being available for seminal plasma. Measurements in duplicate were used to calculate intra-assay variability.

Software STATISTICA Cz, version 6, StatSoft, Inc. (2001) was used for data analysis. The non-parametric Mann-Whitney U-test and Spearman rank correlation for evaluation of TAS levels in seminal plasma and blood serum.

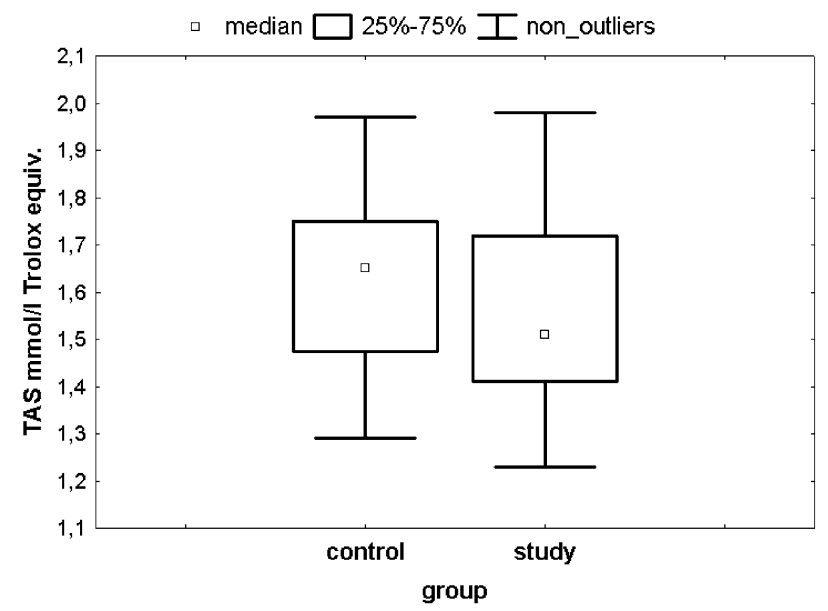

Fig 3. Difference between blood serum TAS levels in the control and study groups. 


\section{RESULTS}

The study group included samples of 38 males aged $24-40$ years $(31.7 \pm 4.3)$ where only 14 males were normospermic and 24 males had mostly combined pathologies. The values (mean $\pm \mathrm{SD}$ ) for sperm parameters in the study group were $51 \pm 33 \times 10^{6} / \mathrm{ml}$ for sperm concentration, $44 \pm 12 \%$ for sperm motility and $26 \pm 12 \%$ for sperm morphology.

The control group included samples of 24 normospermic healthy volunteers aged $21-40$ years $(28.4 \pm 6.2)$. The values (mean $\pm \mathrm{SD}$ ) for sperm parameters in the control group were $58 \pm 36 \times 10^{6} / \mathrm{ml}$ for sperm concentration, 57 $\pm 5 \%$ for sperm motility and $35 \pm 5 \%$ for sperm morphology.

The ROS production in semen varied from undetectable levels to $345.000 \mathrm{RLU} / \mathrm{min} / 2.10^{7}$ spermatozoa in the control group and to a much greater extent, from undetectable levels up to 17 million RLU/min/2.10 ${ }^{7}$ spermatozoa in the study group, but the medians for ROS production were similar in study group (4850 RLU/min) and control group (5450 RLU/min). Fig 1 shows the overlapping histograms in both groups. The ROS production is expressed as $\log _{10}(\mathrm{ROS}+1)$.

The values (mean $\pm \mathrm{SD}$, range, median) for TAS in seminal plasma in the study group were significantly lower $(\mathrm{p}<0.02)$ than in the control group $(2.15 \pm 0.29$ versus $2.32 \pm 0.33 ; 1.63-2.64$ versus $1.45-2.66 ; 2.11$ versus 2.48, resp.). See Fig 2.

Serum TAS levels were also lower in the study group compared to the control group ( $1.58 \pm 0.20$ versus 1.61 $\pm 0.19 ; 1.23-1.98$ versus $1.29-1.97 ; 1.51$ versus 1.65$)$ but the difference was not significant (Fig 3).

The TAS in seminal plasma was on average 1.4 times higher than in serum. The loose positive correlation between seminal plasma and serum TAS in paired samples from subjects from the study group was statistically significant $(r=0.479, p<0.05)$ but individual serum values were not predictive of corresponding seminal plasma values. Similar correlation between seminal plasma and serum TAS was detected in the control group but without significance probably due to a smaller number of samples in this group.

The lowest mean TAS levels in seminal plasma $(2.00 \pm 0.17 \mathrm{mmol} / 1)$ as well as in blood serum $(1.50 \pm$ $0.09 \mathrm{mmol} / \mathrm{l}$ ) in this study was found in samples from 5 males from infertile couples with the highest sperm ROS production.

The intra-assay coefficient of variation (cv) for the seminal plasma sample was $2.7 \%$ and for the blood serum $1.9 \%$.

\section{DISCUSSION}

A systematic review of publications ${ }^{2}$ that evaluate oxidative stress in semen from 1966-2003 concluded that the literature as a whole provides contradictory findings and that it is necessary to carry out more work to identify all the enzymatic and non-enzymatic systems involved in oxidative stress in sperm.

The original enhanced chemiluminescence methods to measure TAC in seminal plasma which detect non-enzymatic antioxidants are sensitive, but they require a strict control of assay conditions. This limits their introduction into clinical practice. The newer reports of seminal plasma antioxidant capacity determination by the spectrophotometric method using ABTS reaction are few and described for a limited number of subjects. Accordingly, there is no consensus on reference total antioxidant capacity of seminal plasma in healthy fertile men. One Italian group ${ }^{16,17}$ using TAS Randox ${ }^{\circledR}$ reported in 25 controls mean seminal plasma TAS levels of $1.21 \pm 0.16 \mathrm{mmol} / 1$ and in another 18 control subjects median TAS 1.19 with a range from 1.0 to $1.4 \mathrm{mmol} / \mathrm{l}$, resp. Higher mean TAS level of 2.02 $\pm 0.16 \mathrm{mmol} / 1$ has been described in 20 fertile Turkish men $^{18}$. Neither of the above papers specified details of their procedure. Our reference values for seminal plasma derived from 24 healthy normospermic volunteers $(2.32 \pm$ $0.33 \mathrm{mmol} / \mathrm{l})$ are so far the highest reported. It is difficult to speculate about the reason for the differences including by e.g. different population, manual or automated version of TAS Randox ${ }^{\circledR}$ kit, seminal plasma viscosity.

In spite of differences in reference values, lower seminal plasma antioxidant capacity was found in human semen with oligoasthenospermia ${ }^{16}$ or with astheno- and asthenoteratospermi $a^{18}$. Also our study showed significantly lower $(p<0.02)$ TAS levels in seminal plasma in the study group of men from infertile couples with a prevalence of pathological spermiogram.

The lowest mean TAS levels in seminal plasma (2.00 $\pm 0.17 \mathrm{mmol} / \mathrm{l}$ ) in this study was found in samples from 5 males from infertile couples with the highest sperm ROS production. This fact may be a reflection of the reciprocal relationship between the two extremes of the oxido-reductive balance in the ejaculate.

Our pilot study suggests that TAS Randox ${ }^{\circledR}$ kit may be used in clinical studies intended to identify decreased antioxidant power in seminal fluid of infertile men.

\section{ACKNOWLEDGMENT}

The study was supported by the grant of Internal Grant Agency of the Czech Ministry of Health No. 1A8621-5

\section{REFERENCES}

1. Agarwal A, Nallella KP, Allamaneni SS, Said TM. Role of antioxidants in treatment of male infertility: an overview of the literature. Reprod Biomed Online 2004; 8(6):616-27.

2. Garrido N, Meseguer M, Simon C, Pellicer A, Remohi J. Pro-oxidative and anti-oxidative imbalance in human semen and its relation with male fertility. Asian J Androl 2004; 6(1):59-65.

3. Whitehead TP, Thorpe GHG, Maxwell SRJ. Enhanced chemiluminescent assay for antioxidant capacity in biological fluids. Analytica Chimica Acta 1992; 266:265-77. 
4. Benzie IF, Strain JJ. The ferric reducing ability of plasma (FRAP) as a measure of "antioxidant power": the FRAP assay. Anal Biochem 1996; 239(1):70-76.

5. Apak R, Guclu K, Ozyurek M, Karademir SE, Altun M. Total antioxidant capacity assay of human serum using copper(II)-neocuproine as chromogenic oxidant: the CUPRAC method. Free Radic Res 2005; 39(9):949-61.

6. Rice-Evans C, Miller NJ. Total antioxidant status in plasma and body fluids. Methods Enzymol 1994; 234:279-293.

7. Cao G, Prior RL. The measurement of oxygen radical absorbance capacity in biological samples. Methods Enzymol 1999; 299:5062.

8. Ziyatdinova GK, Budnikov HC, Pogorel'tzev VI. Electrochemical determination of the total antioxidant capacity of human plasma. Anal Bioanal Chem. 2005; 381(8):1546-51.

9. Firuzi O, Lacanna A, Petrucci R, Marrosu G, Saso L. Evaluation of the antioxidant activity of flavonoids by "ferric reducing antioxidant power" assay and cyclic voltammetry. Biochimica et Biophysica Acta 2005 ; 1721:174-184

10. Rohn S, Kroh LW. Electron spin resonance - a spectroscopic method for determining the antioxidative activity. Mol Nutr Food Res 2005; 49(10):898-907.

11. Aitken RJ. A free radical theory of male infertility. Reprod Fertil Dev 1994; 6(1):19-23.

12. Kolettis PN, Sharma RK, Pasqualotto FF, Nelson D, Thomas AJ
Jr, Agarwal A. Effect of seminal oxidative stress on fertility after vasectomy reversal. Fertil Steril 1999; 71(2):249-55

13. Said TM, Kattal N, Sharma RK, Sikka SC, Thomas AJ Jr, Mascha E, Agarwal A. Enhanced Chemiluminescence Assay vs Colorimetric Assay for Measurement of the Total Antioxidant Capacity of Human Seminal Plasma. Journal of Andrology 2003; 24(5):676-680.

14. WHO laboratory manual for examination of human semen and sperm-cervical mucus interaction. $4^{\text {th }}$ ed.Cambridge University Press, New York, 1999. p. 34-35

15. Novotny J, Oborna I, Brezinova J, Svobodova M, Hrbac J, Fingerova $\mathrm{H}$. The occurrence of reactive oxygen species in the semen of males from infertile couples. Biomed Pap Med Fac Univ Palacky Olomouc Czech Rep. 2003; 147(2):173-6.

16. Siciliano L, Tarantino P, Longobardi F, Rago V, De Stefano C, Carpino A. Impaired seminal antioxidant capacity in human semen with hyperviscosity or oligoasthenozoospermia. J Androl 2001; 22:798-803.

17. Carpino A, Tarantino P, Rago V, De Sanctis V, Siciliano L. Antioxidant capacity in seminal plasma of transfusion-dependent beta-thalassemic patients. Exp Clin Endocrinol Diabetes 2004; 112(3):131-4.

18. Koca Y, Ozdal OL, Celik M, Unal S, Balaban N. Antioxidant activity of seminal plasma in fertile and infertile men. Arch Androl 2003; 49(5):355-9. 